\title{
Steered molecular dynamics investigations of protein function
}

\author{
Barry Isralewitz, Jerome Baudry, Justin Gullingsrud, \\ Dorina Kosztin, and Klaus Schulten
}

\author{
Beckman Institute for Advanced Science and Technology, University of Illinois at \\ Urbana-Champaign, Urbana, IL, USA
}

\begin{abstract}
Molecular recognition and mechanical properties of proteins govern molecular processes in the cell that can cause disease and can be targeted for drug design. Single molecule measurement techniques have greatly advanced knowledge but cannot resolve enough detail to be interpreted in terms of protein structure. We seek to complement the observations through so-called Steered Molecular Dynamics (SMD) simulations that link directly to experiments and provide atomic-level descriptions of the underlying events. Such a research program has been initiated in our group and has involved, for example, studies of elastic properties of immunoglobulin and fibronectin domains as well as the binding of biotin and avidin. In this article we explain the SMD method and suggest how it can be applied to the function of three systems that are the focus of modern molecular biology research: force transduction by the muscle protein titin and extracellular matrix protein fibronectin, recognition of antibody-antigene pairs, and ion selective conductivity of the $K^{+}$channel. (C) 2001 by Elsevier Science Inc.
\end{abstract}

\section{INTRODUCTION}

Experimental techniques based on the application of mechanical forces to single molecules in small assemblies have been applied to study the binding properties of biomolecules and their response to external mechanical manipulations. Among such techniques are atomic force microscopy (AFM), ${ }^{1}$ optical tweezers, ${ }^{2}$ biomembrane force probe,${ }^{3}$ and surface force apparatus experiments. ${ }^{4}$ These techniques have inspired us and others to adopt a similar approach for the study of biomolecules by means of computer simulations.

Steered Molecular Dynamics (SMD) induces unbinding of ligands and conformational changes in biomolecules on time scales accessible to molecular dynamics simulations. For this

Corresponding author: K. Schulten, Beckman Institute, University of Illinois, Urbana, IL 61801, USA.

E-mail address: kschulte@ks.uiuc.edu purpose, time-dependent external forces are applied to a system and the responses of the system are analyzed. SMD calculations, pioneered in our group, have already provided important qualitative insights into biologically relevant problems for applications ranging from identification of ligand binding pathways $^{5-10}$ to the explanation of elastic properties of proteins. ${ }^{11-14}$ SMD, in particular, has revealed the participation of aminoacid side groups in guiding biotin into its avidin binding site; has discovered the binding path of retinal in bacteriorhodopsin from the lipid phase, rather than the aqueous phase as erroneously believed for decades ${ }^{6}$; and has suggested that ATP hydrolysis in actin ${ }^{7}$ as well as hormone binding in certain nuclear hormone receptors ${ }^{8}$ proceed by a back-door mechanism. The SMD method and closely related work by other groups are described elsewhere. ${ }^{15-20}$

In this study we introduce first the SMD method and suggest three exemplary SMD studies for future investigations: a simulation of force transduction by the muscle protein titin and the extracellular matrix protein fibronectin, a simulation of antibody-antigene binding, and a simulation of ion-selective conduction by an ion channel. We begin by introducing the three latter systems.

\section{Stretching of Mechanical Proteins}

Proteins need to fold efficiently into their native conformations to carry out their functions, but also need to avoid aggregation of metastable folding intermediates. ${ }^{21,22}$ Traditionally, experiments and modeling have focused on the folding and unfolding behavior of proteins in response to temperature changes and to chemical perturbations through $\mathrm{pH}$ changes or addition of denaturants. ${ }^{23-32}$ Another factor that can lead to unfolding of proteins is mechanical stress, in particular, stretching. ${ }^{33-35}$ Such stretching arises in muscle, in the extracellular matrix, and at cell receptors during cell motion. We refer to proteins that are designed to respond to stretching under physiological conditions as mechanical proteins.

These proteins often contain multiple domains linked together in a linear sequence. The response of mechanical proteins to external force can be studied by investigating the individual domain's response to stretching. Domains arising in 
several systems have evolved to withstand certain levels of mechanical force ${ }^{36,37,33}$ as well as to sense the application of mechanical strain. ${ }^{12}$ One example is presented by the domains of titin, a giant protein that provides muscle elasticity ${ }^{38,39}$ and controls chromosome shape ${ }^{40}$; the elasticity of titin is due in part to its 300 immunoglobulin (Ig) and fibronectin type III (FnIII) domains. Understanding mechanical proteins can lead to advances in a number of medically relevant areas, including cardiac muscle function, cancer metastasis, and functioning of the extracellular matrix and connective tissues.

Atomic force microscopy (AFM) ${ }^{33,36,37}$ and optical tweezer experiments ${ }^{34,35}$ stretch individual proteins and measure the extensions of proteins as a function of applied force. AFM experiments demonstrate that Ig and FnIII domains behave like elements of a linearly jointed entropic spring and exhibit sawtooth patterns in the measured force-extension profiles. The value of the force peaks in these patterns is about $200 \mathrm{pN}$. From the data one can conclude that connected Ig/FnIII domains unfold one-by-one and that each domain is protected against stretching and unfolding by an energy barrier of about 22 $\mathrm{kcal} / \mathrm{mol} .{ }^{33}$ Similar AFM experiments that stretch proteins consisting of helical domains show much lower force peaks in their corresponding force-extension curves, indicating muchreduced protection against stretch-induced unfolding. ${ }^{41}$

One would like to understand how the architecture of $\beta$-sandwich domains has served nature to design proteins that can withstand high stretch forces and that can act as stress sensors as in the case of FnIII domains. ${ }^{12}$ One would also like to resolve at the atomic level the mechanical stretching and unfolding of protein domains, a resolution that is still out of reach of experiment. SMD simulations ${ }^{15,16,42}$ are ideally suited to address the respective questions about structure-function relationships of Ig and FnIII domains. Indeed, our already completed SMD studies, published earlier ${ }^{1-14}$ and presented below, which stretched single protein domains accounting explicitly for solvent, qualitatively reproduced the AFM force-extension profile. ${ }^{11,14}$ Analysis of simulation trajectories suggests that backbone hydrogen bonding between $\beta$ strands protects the Ig and FnIII domains against stretching and unfolding.

Several theoretical groups have also published studies on forced unfolding of protein domains, employing approaches and methods different from those applied in our group. Rohs et al. ${ }^{43}$ studied the stretching of $\alpha$-helix and $\beta$-hairpin systems using molecular mechanics; they estimated the magnitude of forces involved in the unfolding of these secondary protein structures. Socci et al. ${ }^{44}$ studied the relation between force dependence and the reaction coordinate by stretching a lattice model. Evans and Ritchie ${ }^{45}$ modeled the Ig domain unfolding as a single bond-breaking event and approached the problem using Kramers-Smoluchowski theory.

Paci and Karplus ${ }^{20}$ studied FnIII unfolding by means of biased molecular dynamics using an implicit solvent model to reduce computational effort; the authors, extremely confident in their solvent model, suggested that the dominant barrier against unfolding is due to vdW interactions, and not due to hydrogen bonds. The critique of these authors of our first round of simulations ${ }^{12}$ had been based on a factor of 10 difference between calculated and observed forces, claiming that at the high speed of stretching, we overestimated hydrogen bond forces. However, we have since reduced the speed of stretching ${ }^{14}$ and our calculated force converges within a factor of two of observation; the improved simulations revealed in all cases the same hydrogen bond-breaking scenario as in the earlier work, arguing strongly for hydrogen-bond protection.

Our goal in modeling mechanical proteins is to obtain an atomic-level view of the process of forced domain unfolding. We want to accomplish this by incorporating into simulations two known properties of the system: the experimentally derived static structure and the experimentally known forceextension curve. In achieving this goal, our theoretical model should meet two criteria: first, the model should correspond closely to the experimental (AFM) situation, to provide a nonambiguous check on the model's validity; second, the results should provide information on the process at atomic-level resolution that cannot be obtained from experiment. The works by Rohs et al. and by Paci and Karplus both involve atomiclevel detail; however, both use stretching protocols that are much different from the AFM and optical tweezer experiments that have been performed so far, and thus are difficult to compare with existing measurements. The models used by Socci et al. and by Evans and Ritchie do not fulfill the second criterion; both are a drastic abstraction of the actual process, i.e., involving a lattice model and an unfolding potential along a single degree of freedom, respectively, and thus do not provide atomic-level detail.

The SMD method can satisfy both criteria listed above. Force-extension curves produced by simulations can be directly compared with AFM data to check validity. The SMD trajectories account for the unfolding process at atomic-level detail and, hence, reveal structure-function relationships for protein elasticity properties. The proposed work will clarify the differences among the different theoretical approaches employed elsewhere ${ }^{11-14,20,43,44,45}$ and provide a means to unite these approaches, while relating them to experiment.

\section{Antibody-Antigen Interactions}

Antibodies are proteins produced by the immune response. Antibodies are selective, high-affinity receptors for a seemingly unlimited number of antigenic molecules that threaten normal cellular function. This diversity in antigen recognition can be utilized to produce what are known as "catalytic" antibodies. These catalytic antibodies are capable of performing specific chemical reactions on their antigenic substrates, making these antibodies therapeutic candidates, since they would be able to catalyze clinically relevant chemical reactions within the body. Understanding the structure and function of catalytic antibodies could lead to targeted development of drug molecules that may be used, for example, in cancer therapy or drug addiction treatment. ${ }^{46}$

The immune response is an intriguing defense mechanism unique to eukaryotic organisms. The molecular basis of this mechanism is not totally understood. After first exposure of the antibody to a specific antigen, somatic hypermutation of antibody genes leads to the expression of a mature, mutated, antibody. ${ }^{47}$ The mature antibody exhibits an increased affinity for its antigen several orders of magnitude over that of the germline (wildtype) antibody. As a result of this maturation process the antibody becomes more efficient in recognizing and distinguishing its antigenic substrate. Enhancement of antigen specificity and of kinetic factors have been demonstrated in a series of papers on the esterolytic antibody $48 \mathrm{G} 7.48,49,50$ Crystal structures of the Fab fragments (the antigen binding fragments) of the catalytic antibody $48 \mathrm{G} 7$, germline or mature, 
complexed with a hapten (a transition state analog of the antigen, that does not experience chemical reaction, Figure 1) or without hapten, were recently obtained by Wedemayer et al. ${ }^{50}$

Analysis of the crystal structures showed that the mature antibody differs from the germline by nine somatic mutations, three in the light chain and six in the heavy chain. These mutations are not all located close to the antigen binding site: two of the substitutions were within $5.5 \AA$ of the hapten, the other seven mutations were within $10 \AA$ and $15 \AA$ (Figure 1). Improvements in hapten-binding affinity result from somatic mutations that are distant from the antigen-binding site.

The crystal structures suggest that certain residues are playing a role in stabilizing the antibody and strengthen antibodyhapten interactions. Wedemayer et al. ${ }^{50}$ list differences between the bound and unbound forms of the crystal structures in the case of the germline antibody. The authors observed an increase in the number of hydrogen bonds as well as changes in the relative orientation of the light and heavy variable chains of $4.6^{\circ}$. They also observed that certain tyrosine residues located near the surface of the antibody (residues $\mathrm{Tyr}^{H 33}$ and $\mathrm{Tyr}^{H 98}$ ) undergo structural rearrangement, leading to an enhancement of the $\pi$-stacking interactions between antibody residues $\mathrm{Tyr}^{H 98}, \mathrm{Tyr}^{H 99}$ and $\mathrm{Arg}^{L 46}$ (the $\mathrm{H}(\mathrm{L})$ indices refer to heavy (light) chains). Antibody-hapten interactions are modified as well by formation of a packing interaction between the aliphatic tail of the hapten and residue $\mathrm{Tyr}^{H 98}$.

According to Wedemayer et al., ${ }^{50}$ hapten binding on the germline antibody leads to significant structural rearrangements, whereas binding of the hapten on the mature antibody does not. The important point here is that equivalent structural changes can be achieved either by maturation of the antibody or by hapten binding. SMD simulations may investigate how these two different events lead to the observed convergence of structures. The Wedemayer et al. study suggests that the germline antibodies can adopt more isoenergetic conformations than the mature antibody. Germline antibodies, having more conformational flexibility, bind antigen with an "induced fit" mechanism, while binding of the hapten by the mature antibody operates through a "lock-and-key" mechanism. This "conformational hypothesis" could explain differences in affinity between the germline and mature forms of the antibody, since the mature antibody would be preadapted for the acquisition of its catalytic function.

Molecular dynamics provides a direct approach to study these structural issues. In fact, the method has been used to test changes in flexibility for other antibody systems. ${ }^{52,53}$ These earlier studies confirmed the possibility that antibody maturation or antigen unbinding change the flexibility of the antibody. However, the accuracy of the investigations was limited by the absence of crystal structures for both the unbound antibody and its mature form. These limitations can now be overcome by the availability of crystal structures for all of the above antibody states, i.e., germline bound/unbound and mature bound/unbound..$^{50}$

One objective of an SMD study should be to test the conformational hypothesis by quantifying changes in protein flexibility upon binding of the hapten. One can determine if the mature antibody, even in its unbound state, has a reduced flexibility over that of the germline. This result would confirm<smiles>O=C([O-])CCCC[C@@H](O)Oc1ccc([N+](=O)[O-])cc1</smiles><smiles>O=C([O-])CCCCC([O-])O</smiles><smiles>O=C([O-])CCCCP(=O)([O-])Oc1ccc([N+](=O)[O-])cc1</smiles>

(a)

(b)

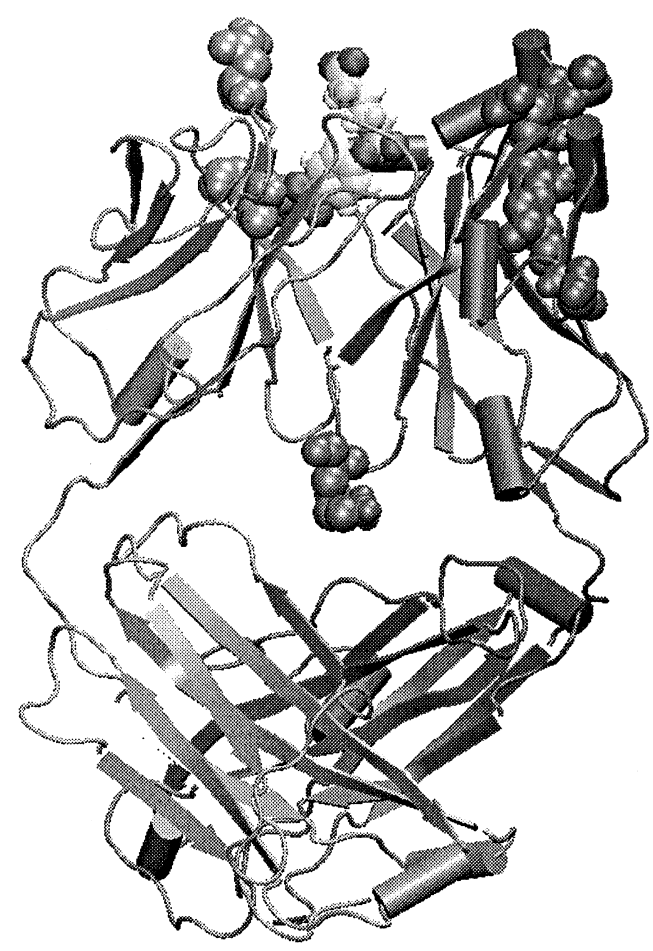

Figure 1. (left) Chemical structures of: (a) substrate of $48 G 7$ antibody, (b) product of the enzymatic reaction, (c) hapten, transition state analog of compound a; (right) Cartoon representation of the crystal structure of the Fab fragment of mutant antibody $48 G 7$ bound to hapten. Mutated residues are represented as dark spheres. Hapten atoms are represented as light-colored spheres. Figure created using $V M D^{51}$. 
the hypothesis drawn on the basis of Wedemeyer et al.'s study. ${ }^{50}$ For the purpose of such study one can employ the SMD method to pull the hapten from its binding site and perturb the equilibrated structures built from the crystal structures, recording the response of the protein to the perturbation and monitoring how several structural descriptors vary with time.

Other objectives could be to address more specifically the energetics and thermodynamics of the binding process itself. A detailed analysis of the kinetics of binding of the antigen and of the hapten has been given elsewhere. ${ }^{48}$ It has been shown that the dissociation constant $k_{\text {off }}$ of the hapten (equivalent to the $k_{\text {cat }}$ measured for antigen-antibody enzymatic reaction) goes from a value higher than $1 \mathrm{~s}^{-1}$ in the case of the germline antibody, to a value of $1.41 \times 10^{-3} \mathrm{~s}^{-1}$ in the case of the mature antibody. These parameters indicate that the kinetics of hapten unbinding are slower for the mature antibody, suggesting a higher free energy barrier on the unbinding pathway of the hapten. These kinetic data suggest SMD simulations that distinguish between the unbinding pathways of the mature and germline antibodies, and that quantify the relative importance of hapten-antibody interactions existing on the unbinding pathways.

Finally, one could simulate the unbinding of the substrate and unbinding of the product species of the enzymatic reaction. Specifically, one needs to understand structurally how the catalytic antibody is able to recognize its antigenic substrate, bind it, undergo catalysis, and release the enzymatic endproduct. Specific residues may be identified that interact preferentially with either the substrate or the product molecules. This will provide insight into the structural basis of the distinction by the antibody between product and substrate in the enzymatic reaction. SMD investigations also provide the opportunity to address in more structural detail the role of antibody maturation in antibody-antigen interactions.

\section{Conduction of Ions Through Channels}

Ion channels are membrane-spanning proteins that form a pathway for the flux of inorganic ions across cell membranes. ${ }^{54}$ Diffusion of ions through the ion channels seems to be a simple physical process, and yet the ion channels are responsible for all electrical signaling in biology. Among their many functions, ion channels regulate the secretion of hormones into the bloodstream, generate the electrical impulses underlying information transfer in the nervous system, and control the pace of the heart and of the muscle system. ${ }^{54}$ To understand the physical basis of ion channel conduction, one needs to characterize the channel's structural and dynamic properties.

Potassium ions diffuse across the cell membrane through proteins called $\mathrm{K}^{+}$channels. Recently, the crystal structure of the $\mathrm{K}^{+}$channel from Streptomyces lividans (KcsA channel) was resolved. ${ }^{55}$ The structure revealed that the KcsA channel is formed by four identical subunits, each subunit containing two $\alpha$-helices connected by an approximately 30 -amino acid-long loop protruding into the pore region. The pore region is approximately $45 \AA$ long and consists of an inner pore (starting from inside the cell), a large cavity near the middle of the pore, and the so-called selectivity filter that separates the cavity from the extracellular solution. The inner pore and the internal cavity are hydrophobic regions; in contrast, the selectivity filter is lined exclusively by polar main-chain atoms belonging to the so-called "signature sequence." Experimental studies showed that this "signature sequence," which comprises an eightamino-acid sequence motif, is responsible for potassium selectivity. ${ }^{56}$ The obvious implication is that these amino acids form a special structure, used uniquely by potassium channels to coordinate potassium ions in a selective manner. ${ }^{55,56}$

How does the selectivity filter work? The authors of the crystal structure of the KcsA channel ${ }^{55}$ propose that the selectivity filter attracts and concentrates $\mathrm{K}^{+}$ions. The presence of multiple ions in the selectivity filter results in mutual repulsion and, thereby, allows conduction to occur. The selectivity filter accommodates perfectly well a dehydrated $\mathrm{K}^{+}$ion, but not an $\mathrm{Na}^{+}$ion that has a smaller radius.

The essential feature of these channels is their ion selectivity, the discriminating nature of the ion conduction pathway. Advances made using structural and electrophysiological techniques have raised a new set of questions about the precise molecular details of ion selectivity. What is the chemical basis for the impressive fidelity with which the channel distinguishes between $\mathrm{K}^{+}$and $\mathrm{Na}^{+}$ions? How does the signature sequence encode a potassium selectivity filter? These questions are fundamentally structural in nature: therefore one has to "see" potassium ions coordinated at their binding sites in the pore before one can understand and explain ion selectivity in a potassium channel.

One of the best tools for making the connection between the structure and function of ion channels is molecular dynamics (MD) simulation. MD simulations allow one to follow the conformational changes in the structure, and the movement of $\mathrm{K}^{+}$ions across the potassium channel, by modeling the bonded and nonbonded interactions between atoms using simple classical potentials. In the case of ion diffusion across the channel, no chemical bonds are formed and the use of classical mechanics in all parts of the simulation is an acceptable approximation. The availability of the crystal structure of the $\mathrm{K}^{+}$channel from Streptomyces lividans (KcsA channel) ${ }^{55}$ opens a new avenue for understanding the energetics of fundamental processes such as ion binding and ion selectivity by using computer simulations. Unfortunately, full atomistic simulations of ion channels embedded in a lipid membrane have to account for thousands of atoms and the time scale of the permeation of even one single ion, of approximately $1 \mu \mathrm{s}$, is inaccessible to molecular dynamics simulations at present. The extraordinary effort to carry out ns simulations, ${ }^{57,58}$ or of a single $\mu$ s simulation, ${ }^{24}$ underscores the point. The difficulty in simulating the passing of $\mathrm{K}^{+}$or $\mathrm{Na}^{+}$ions through the channel can be overcome by using SMD, which "speeds up" a process. One can use SMD of the $\mathrm{K}^{+}$channel to study the passing of $\mathrm{K}^{+}$and $\mathrm{Na}^{+}$ions through the channel. Trajectories obtained from the SMD simulations will allow one to determine the potential of mean force in both cases. Such simulations may, for example, test the hypothesis that the selectivity filter frees the $\mathrm{K}^{+}$ion from its hydration shell more effectively than the $\mathrm{Na}^{+}$ion. This should be reflected in the potential of mean force of $\mathrm{K}^{+}$and $\mathrm{Na}^{+}$ions along the axis of the channel, calculated by employing the time series analysis method in Gullingsrud et al. ${ }^{59}$

\section{THE SMD METHOD}

SMD is a novel approach to the study of the dynamics of binding-unbinding events in biomolecular systems and of their elastic properties, reviewed elsewhere. ${ }^{15}$ The simulations re- 
veal the details of molecular interactions in the course of unbinding ${ }^{5-10}$ or unfolding, ${ }^{11-14}$ thereby providing important information about the molecular mechanisms underlying these processes. The advantage of SMD over conventional MD is the possibility of inducing relatively large conformational changes in molecules on ns time scales accessible to actual computations.

SMD is closely related to the well-known umbrella sampling and free energy perturbation methods, ${ }^{60}$ as well as the weighted histogram analysis method ${ }^{61}$ that seek to determine energy landscapes. The latter methods typically involve only small conformational changes or changes of chemical constituents. Since the analysis of respective simulations assumes a quasiequilibrium process, computations need to enforce changes sufficiently slowly to achieve accurate results and, hence, require extensive computer resources.

\section{Why SMD?}

SMD simulations, like umbrella sampling, superimpose a timedependent force on simulated biopolymers that pull the systems along certain degrees of freedom. SMD simulations are equivalent to umbrella sampling when applied forces are weak, change very slowly in time, and induce minor overall changes. Of interest here is the opposite limit, when major changes are induced, e.g., a ligand is extracted from an enzyme or a protein's termini are stretched to initiate unfolding, and when superimposed forces change rapidly in time, leading to significant deviations from equilibrium. This is the limit that one is supposed to avoid in the umbrella sampling, free energy perturbation theory, and weighted histogram analysis methods, but that one seeks to exploit in SMD, expecting of course certain limitations. There are several reasons why this seems possible, and indeed wise.

First, one can assume in the analysis of simulation results from the outset that systems described are not at equilibrium, and employ nonequilibrium descriptions for the analysis. That this is possible in principle has been shown in the work of Jarzynski, who demonstrated that free energy differences can be obtained through exponential averages of irreversible work. $^{62,63}$ Our group demonstrated ${ }^{64}$ that one can construct potentials of mean force from SMD trajectories and we derived an error bound on this construction that is proportional to the irreversible work done. We carried this construction further, ${ }^{59}$ introducing a time series analysis method based on the Onsager-Machlup function, compared results to those obtained using the weighted histogram analysis method, and showed through examples that potentials of mean force can be reconstructed accurately from multiple SMD runs. The time series analysis method relies sensitively on an accurate determination of friction, a problem that we seek to overcome at present. Application of SMD to the $\mathrm{K}^{+}$channel requires a quantitative analysis of the potential of mean force for ion conduction.

A second argument for the SMD method is that one often seeks not quantitative results, but qualitative answers, i.e., simulations can reveal valuable information even for large error bars. Examples are studies in which binding pathways are identified, i.e., a lipophilic route of retinal binding in bacteriorhodopsin ${ }^{6}$ or back-door mechanisms in actin and nuclear hormone receptors. ${ }^{7,8}$ The key issue of modeling mechanical proteins is the qualitative nature of the protection mechanisms, concerted hydrogen bonds, or van der Waals interactions sta- bilizing the hydrophobic core. The planned SMD investigation of antibodies involves also an essential qualitative component, the identification of amino-acid side groups controlling shape fluctuations of the antigen binding site.

A third, very strong argument for SMD simulations is that they mimic directly AFM experiments and, hence, permit comparison of simulated and observed force-extension profiles, as stressed already above. Lu and Schulten ${ }^{14}$ have documented a convergence of simulation and observation that is further supported by experiment. ${ }^{65}$

\section{How Does SMD Work?}

One way to apply external forces to a protein-ligand complex is to restrain the ligand to a point in space (restraint point) by an external, e.g., harmonic, potential. The restraint point is then shifted in a chosen direction ${ }^{8-10,16,18}$ forcing the ligand to move from its initial position in the protein and allowing the ligand to explore new contacts along its unbinding path. Assuming a single reaction coordinate $x$, and an external potential $U=$ $K\left(x-x_{0}-v t\right)^{2} / 2$, where $K$ is the stiffness of the restraint, and $x_{0}$ is the initial position of the restraint point moving with a constant velocity, $v$, the external force exerted on the system can be expressed as

$$
F=K\left(x_{0}+v t-x\right) .
$$

This force corresponds to a molecule being pulled by a harmonic spring of stiffness $K$ with its end moving with velocity $v$. Alternatively, a fixed restraint point at a distance much larger than the length of the unbinding pathway may be chosen. In this case, the end of the spring does not move and its stiffness is linearly increased with time, ${ }^{5}$ i.e., $K=\alpha t$, and the force is

$$
F=\alpha t\left(x_{0}-x\right)
$$

Other external forces or potentials can also be used, e.g., constant forces or torques applied to parts of a protein to induce rotational motion of its domains. ${ }^{66}$

SMD simulations require selection of a path, i.e., a series of directions of the applied force. In some cases a straight-line path is sufficient, e.g., for avidin-biotin, ${ }^{6}$ actin, ${ }^{66}$ lipids in membranes, ${ }^{9}$ or the unfolding of titin immunoglobulin domains. ${ }^{11,12}$ Other biomolecular systems involve a ligand positioned at the bottom of a convoluted binding cleft, e.g., bacteriorhodopsin, ${ }^{15}$ prostaglandin $\mathrm{H}_{2}$ synthase, ${ }^{10}$ and retinoic acid receptor. ${ }^{8}$ In the latter cases the forced unbinding of the ligand requires the direction of the force to be changed during the simulation to avoid distortion of the surrounding protein. The direction of the force can be chosen randomly ${ }^{67}$ or by guessing a direction on the basis of structural information. ${ }^{8,15} \mathrm{~A}$ force is then applied to the ligand in the chosen direction, and this direction is accepted or rejected based on factors such as conservation of secondary structure of the protein, deformation of the protein, the magnitude of the force applied, the average velocity of the ligand along the unbinding pathway, etc.6,67

\section{Quantitative Information from SMD: Potentials of Mean Force}

In cases where irreversible work done during unbinding can be attributed to a nondispersive frictional force, $\gamma v$, a quantitative 
description of the thermodynamic potentials governing the binding and unbinding processes can be achieved by discounting the irreversible work from the calculated potential of mean force. The error in the reconstructed potentials is related to the irreversible work done on the system and, therefore, may be unacceptably large. The estimate of the friction also presents a challenge, since it can be highly dispersive and may exhibit memory effects. ${ }^{64}$ Irreversibility of the unbinding process can also be accounted for by averaging over an ensemble of SMD trajectories according to the nonequilibrium equality for free energy differences. ${ }^{62,63}$ This approach, however, requires averaging over multiple trajectories, and may be extremely sensitive to insufficient sampling of reaction pathways. Irreversible work might also be discounted by forcing a conformational change in the system followed by the reverse conformational change, i.e., inducing hysteresis. Such an approach may yield a "model free" estimate of the irreversible work component from the hysteresis. ${ }^{68,69}$ Finally, lengthening the simulation time decreases the amount of irreversible work and the simulated process could, ideally, reach quasi-equilibrium in the limit of very long simulation times, approaching free energy perturbation theory. ${ }^{60}$

Is there a practical method to discount the irreversible work done in an SMD simulation and can one determine the potential of mean force that describes the process studied? This seems to be indeed possible. We have proposed recently a method to calculate the potential of mean force (PMF) from SMD simulation. For this purpose we have described and implemented three time-series analysis techniques for reconstructing the PMF from displacement and applied force data gathered from SMD trajectories. ${ }^{59}$ One technique, based on the WHAM theory, ${ }^{70}$ views the unbinding or stretching as a quasi-equilibrium process. The other two techniques assume a Langevin description of the dynamics ${ }^{71}$ to account for the nonequilibrium character of SMD data. One nonequilibrium method, which we refer to as the Gaussian drift method, is based on van Kampen's $\Omega$-expansion72; the second, the "least squares method," is based on a least squares minimization of the Onsager-Machlup action ${ }^{73}$ with respect to the choice of PMF.

The trajectory is modeled as the path of a particle moving in a one-dimensional potential $W(z)$ with a Langevin equation of the form

$$
\gamma \dot{z}=-d W(z) / d z+F(z, t)+\sigma \xi(t) .
$$

In Equation 3, $\gamma$ is the viscous friction coefficient and $\sigma \xi(t)$ represents a stochastic force due to thermal fluctuations in the channel environment. The PMF $W(z)$ determines the dynamics of the ion within the channel, and its calculation will be an important part of any attempt to understand the channel's selectivity.

These analysis methods were tested ${ }^{59}$ on one-dimensional model systems for which the underlying PMF was exactly known. The WHAM method systematically overestimated the potential at large displacements because it made no allowance for the irreversible work done during the simulations.

The Gaussian drift and least squares methods were applied to actual SMD simulation data from a phospholipid membrane monolayer system, depicted in Figure 2. The system and simulation parameters are described elsewhere. ${ }^{9}$ The lipid was pulled by a spring with velocity $v=0.014 \AA / \mathrm{ps}$ and spring constant $k=700 \mathrm{pN} / \AA$. A friction constant of $\gamma=4000 \mathrm{pN}$. ps/A was assumed for both methods, which compares well with the value obtained in other MD simulations, e.g., $5000 \mathrm{pN}$. $\mathrm{ps} / \AA^{18}$ and $2000 \mathrm{pN} \cdot \mathrm{ps} / \AA \mathrm{A}^{16}$

Results of PMF reconstruction using the latter two methods are shown in Figure 2. The reconstructions exhibit several important features. First, the peaks in the applied force (top
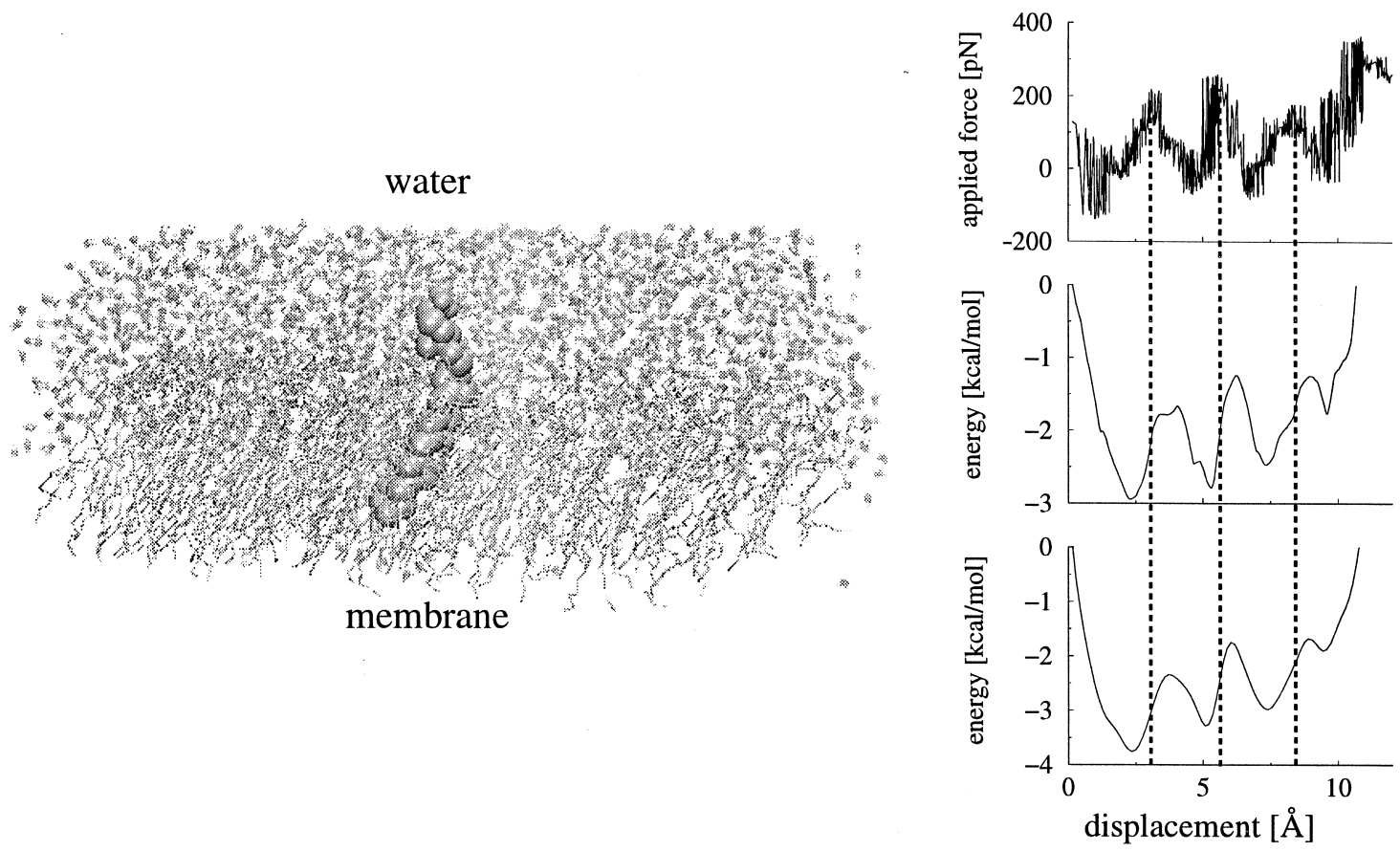

Figure 2. (left) Lipid partially extruded from phospholipid monolayer; (right) Application of PMF reconstruction methods to SMD time series data ${ }^{59}$. 
graph) at $x=3.1,5.6$, and $8.5 \AA$ line up with the uphill slopes of both PMF reconstructions. Second, the height of the barrier at the first peak is approximately $1.5 \mathrm{kcal} / \mathrm{mol}$, an appropriate height value for the breaking of a single hydrogen bond. ${ }^{9}$ Third, the action minimization reconstruction (bottom graph) is essentially identical to the Gaussian drift reconstruction (middle graph).

\section{APPLYING STEERED MOLECULAR DYNAMICS}

We will demonstrate the viability of SMD through three research projects that are ongoing or are presently being initiated in our group. Numerous other projects could have been selected for the same purpose. In fact, our group is often approached with new suggestions for collaborations and we expect that computational modeling will see many exciting applications of SMD being completed over the next years.

\section{Stretching of Mechanical Proteins}

Our research group started modeling protein domain unfolding under mechanical strain in 1997. We were the first group that attempted to investigate this problem in atomic-level detail. ${ }^{11-14}$ We began with a SMD study on forced unfolding of titin immunoglobulin domain I27. ${ }^{11}$ It is noteworthy that Ig and FnIII constitute $\beta$-sandwich domains with $\mathrm{N}$-terminal and C-terminal strands parallel to each other, but pointing in opposite directions. In the natural function of these domains as well as in AFM and optical tweezer experiments, the forces arising stretch apart the termini. The stretching protocols we have used are constant velocity stretching and constant force stretching. The constant velocity stretching simulations of I27 stretching were carried out by fixing the $\mathrm{C}_{\alpha}$ atoms of the first residue (Leu in I27), and by applying external forces to the $\mathrm{C}_{\alpha}$ atom of the last residue harmonically to a restraint point and moving the restraint point with a constant velocity, $v$, in the desired direction. The procedure adopted is equivalent to attaching one end of a harmonic spring to the $\mathrm{C}_{\alpha}$ atom of the last residue and pulling on the other end of the spring. This is similar to the procedure performed on titin and tenascin in AFM experiments, ${ }^{33,36}$ except that the pulling speeds adopted in the simulations are about six orders of magnitude higher than those in the experiment.

The forces experienced by the pulled atom in constant velocity stretching are described in Equation 1. Here $x$ is the position of the pulled atom, $x_{\mathrm{o}}$ is its original position, $v$ is the pulling velocity, $v t$ is the position of the restraining point, and $K$ is the spring constant. The pulling direction was chosen along the vector from the $\mathrm{C}_{\alpha}$ atom of the first residue to the $\mathrm{C}_{\alpha}$ atom of the last residue. We chose for $K$ a value of $10 k_{B} T / \AA^{2}$ with $T=300 \mathrm{~K}$; this choice implies spatial (thermal) fluctuations of the constrained $\mathrm{C}_{\alpha}$ atom of $\delta x=\sqrt{k_{B} T / k}=0.38 \AA$. To realize a movement of the restraint point with nearly constant velocity, the position of the restraint point was changed every 100 fs by $v \Delta t$.

SMD simulations of constant force stretching were implemented by fixing the N-terminus of the domain I27 and by applying a constant force to the $\mathrm{C}$-terminus along the direction connecting the initial positions of $\mathrm{N}$-terminus and $\mathrm{C}$-terminus. The atomic coordinates of the whole system were recorded every ps. For constant velocity stretching, the elongation $d(t)$, defined as the increase of the end-to-end distance between the termini from that of the native fold, was monitored along with the force $F(t)$. For the analysis presented below, often the force was plotted as a function of extension $d$. The $(F(t), d(t)$ graphs are referred to as force-extension profiles. In the case of the constant force stretching, the elongation $d(t)$ was recorded and plotted as $(d(t), t)$, which is referred to as the extension curve.

The recorded force-extension curve qualitatively agrees with the AFM data: we observed a dominant force peak at short extension during the stretching. Detailed simulation trajectory analysis suggest this force peak was caused by the concurrent breakage of a set of backbone hydrogen bonds between $\beta$ strands $\mathrm{A}^{\prime}$ and $\mathrm{G}$ (Figure 3).

We further studied the forced I27 unfolding to connect quantitative results of simulations with data from AFM observations. ${ }^{14}$ We showed that if constant forces stronger than 50 $\mathrm{pN}$ are applied to the terminal ends, the two hydrogen bonds between the antiparallel $\mathrm{A}$ and $\mathrm{B} \beta$-strands break with a concomitant 6-7 $\AA$ elongation of the protein. This is in agreement with a recent AFM observation that an initial elongation of $6 \AA$ in I27 is exhibited when a stretching force of $50-100 \mathrm{pN}$ is applied. ${ }^{65}$ If constant forces strong enough to unfold the domain are applied, the protein is halted after initial extension until the set of all six hydrogen bonds connecting strands $\mathrm{A}^{\prime}$ and $\mathrm{G}$ break simultaneously. We noticed the halt time depended on the value of the force applied to the domain. Even with the same force, the halt time varied with different initial conditions, with the distribution of the halt time agreeing well with a theoretical prediction that accounted for a barrier separating folded and unfolded states. We also demonstrated that SMD simulations that induce unfolding through slow pulling (speed $0.1 \AA / p s$ ) predict unfolding forces that are within a factor of two of force values extrapolated from AFM observations. ${ }^{14}$

We have also studied the unfolding process of FnIII under stretching. ${ }^{12}$ The tenth type III module of fibronectin, FnIII ${ }_{10}$, mediates cell adhesion to surfaces via its integrin binding ${ }^{74}$ motif, $\mathrm{Arg}^{78}, \mathrm{Gly}^{79}$, and $\mathrm{Asp}^{80}$ (RGD), which is placed at the apex of the loop connecting its $\beta$-strands F and G. SMD simulations in which tension was applied to the protein's terminal ends revealed that the $\beta$-strand $\mathrm{G}$ (C-termini strand) is the first to break away from the module upon forced unfolding, while the remaining fold maintains its structural integrity. ${ }^{12}$ The separation of strand $\mathrm{G}$ from the remaining fold resulted in a gradual shortening of the distance between the apex of the RGD-containing loop and the module surface, which potentially reduces the loop's accessibility to surface-bound integrins. ${ }^{75}$ The shortening is followed by a straightening of the RGD loop from a tight $\beta$-turn into a linear conformation which suggests a further decrease of affinity and selectivity to integrins. The RGD loop is therefore strategically located to undergo strong conformational changes in the early stretching stages of the module, and thus constitutes a mechanosensitive control of integrin recognition.

Additionally, we have performed SMD simulations on 10 different protein domains that have not been studied by AFM experiment. ${ }^{13}$ The stretching simulations, each $0.6 \mathrm{~ns}$ in duration, reveal two types of protein responses: the first type, arising in certain $\beta$-sandwich domains, exhibits unfolding only after a force above $1500 \mathrm{pN}$ is applied; the second type, arising in a wider class of protein domain structures, requires significantly weaker forces for unfolding. In the first case, strong 

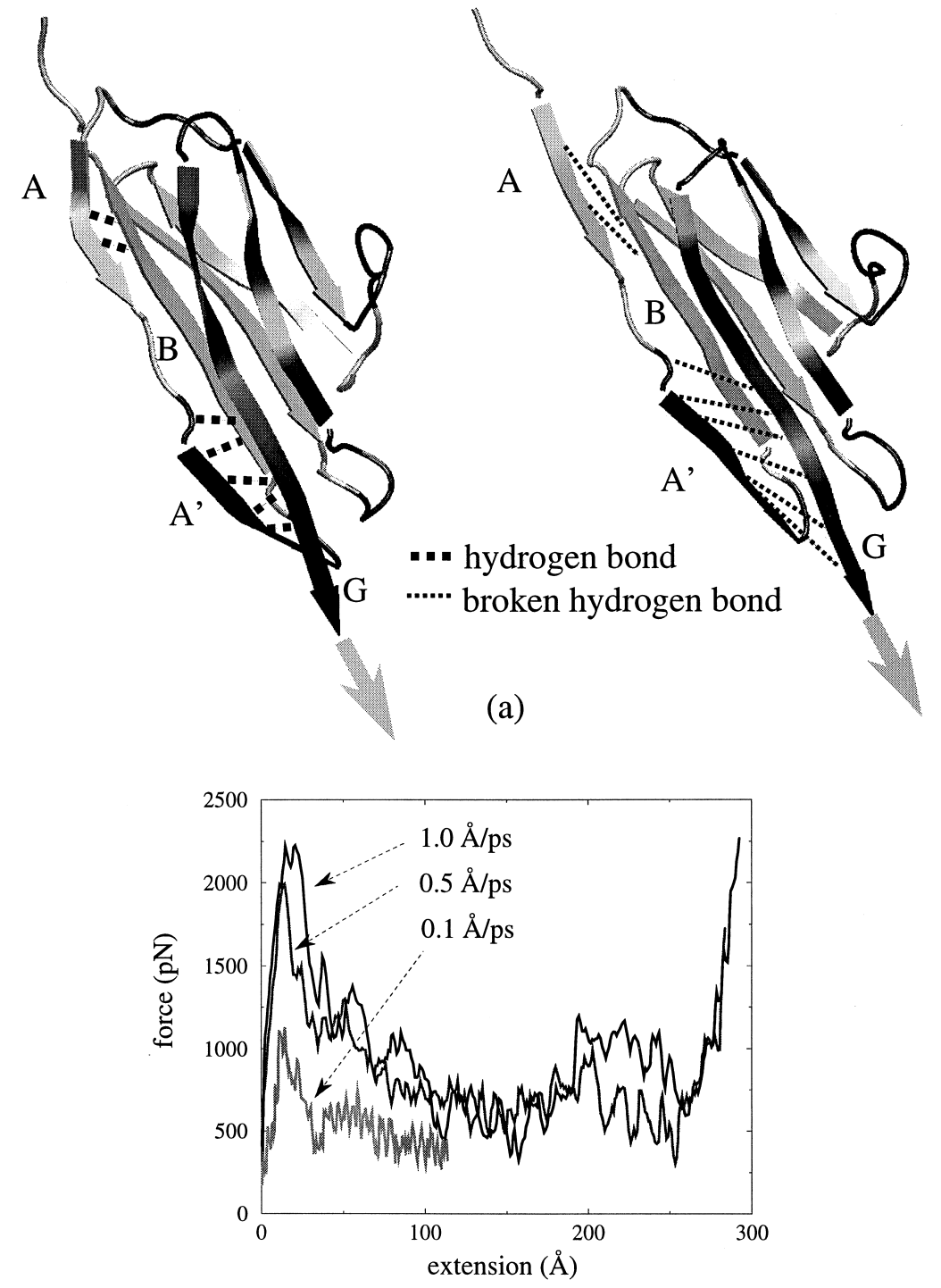

(b)

Figure 3. SMD study of I27 unfolding under mechanical strain. (a) Key steps of I27 unfolding identified by SMD. The domain is drawn in cartoon representation and key hydrogen bonds between strands A-B, and between $A^{\prime}-G$ are shown as dotted lines. Left is a snapshot of $I 27$ extended by $10 \AA$. Right is a snapshot of I27 extended by $17 \AA$. (b) Force extension curves of I27 extended by constant velocity stretching SMD with 3 different velocities.

forces are needed to concertedly break a set of interstrand hydrogen bonds that protect the domains against unfolding through stretching; in the second case, stretching breaks backbone hydrogen bonds stabilizing the structure one-by-one, and does not require strong forces to unfold the domain.

\section{Antibody-Antigen Interactions}

Our preliminary work has focused on building models for studying conformational and dynamical differences of four different crystal structures of the catalytic antibody and its mature form 48G7. The four antibody structures are: (i) germline bound to hapten, (ii) germline unbound, (iii) mature bound to hapten, and (iv) mature unbound.

Our initial goal was to determine if the fluctuations of the germline unbound antibody are larger than the fluctuations of the three other structures of the protein, following the "conformational hypothesis" suggested by the crystal structures. ${ }^{50} \mathrm{We}$ performed semi-empirical calculations to obtain a set of parameters for the antigen molecule-a nitrophenyl phosphonate transition-state analog - to obtain an accurate force field for subsequent molecular dynamics simulations. The four crystal structures were hydrated initially with a thin $(10-\AA)$ hydration shell, leading to a size of $\sim 15,000$ atoms for each of the four hydrated crystal structures. Molecular dynamics simulations of these systems were performed using the CHARMM program ${ }^{76}$ at room temperature for up to $450 \mathrm{ps}$ for each antibody structure. The simulations were run on 8 processors of a Beowulf cluster of Pentium-II processors, taking $\sim 10$ days per simulation of 450ps. The fluctuations of the atoms around their average value for each of the antibodies were determined. We 


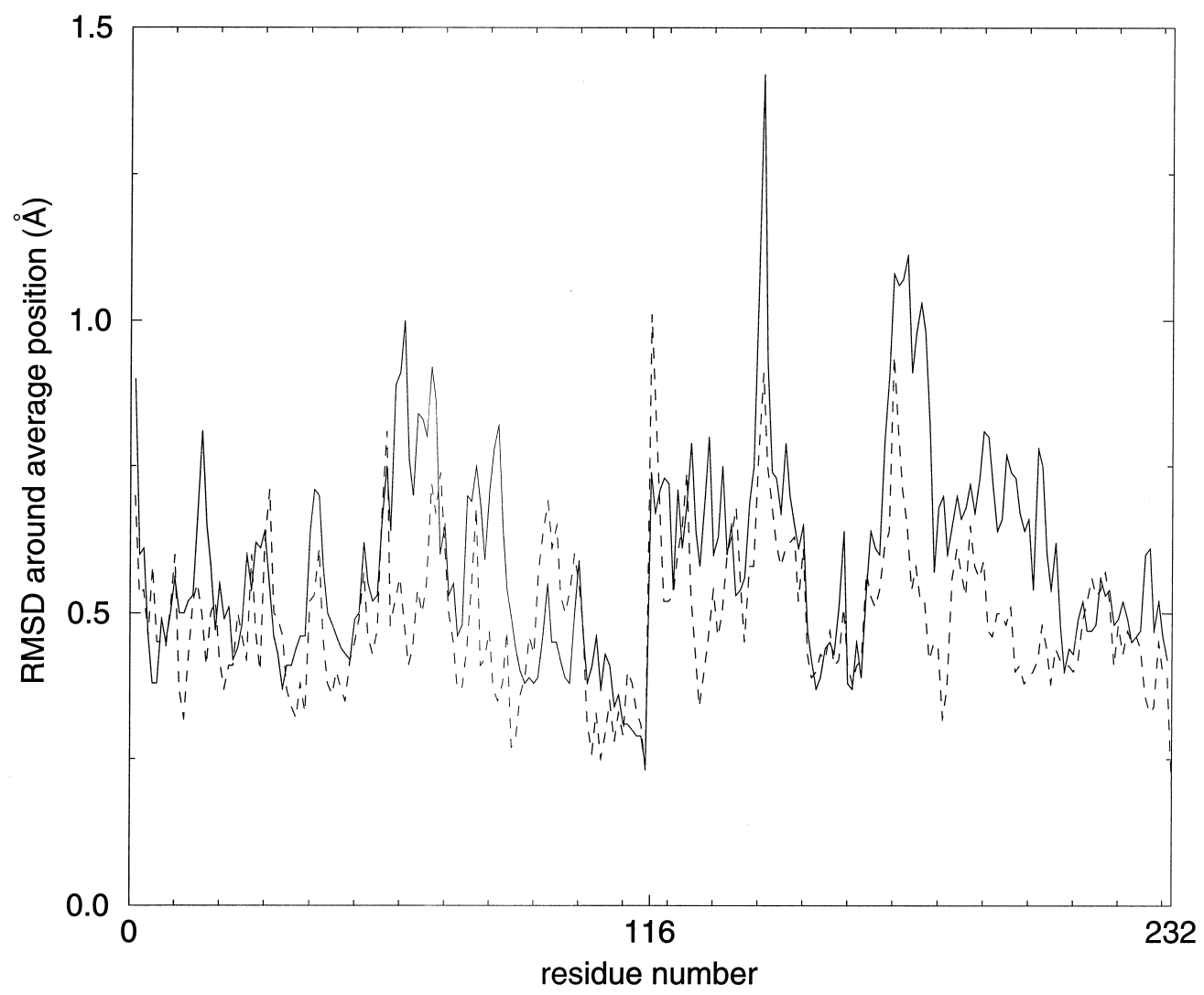

Figure 4. RMSD around average positions of alpha carbons for the variable region of the antibodies, calculated from the first 40 ps of a simulation with thin hydration shell. Residues 1 to 115 are from the light chain, residues from 116 are from the heavy chain. Solid line: germline unbound antibody, dashed line: mature unbound antibody.

observed that the flexibility of the germline antibody is generally higher than that calculated for the mature antibody for short simulation times (see Figure 4).

However, the thin hydration shell used led to a severe distortion of the antibody structure and, therefore, introduced artifacts at long simulation times. A simulation test was then performed with a larger spherical hydration shell, which preserved the antibody structure, but at a higher computational overhead (a molecular size of more than 40,000 atoms per system). Simulations of an antibody with a large hydration shell, using a switching cutoff function between $10 \AA$ and $12 \AA$ for nonbonded interactions, with an integration time step of 1 fs, took $\sim 14$ days for 500-ps simulation time on 8 processors of a Pentium-II cluster, employing our group's NAMD2 program. ${ }^{77}$ From these preliminary results we conclude that a more realistic hydration model of the crystal structures will allow us to quantify in a reliable way a structural change over long periods of simulation. New molecular dynamics simulations will be performed on these antibodies with a larger hydration shell. The structure thus modeled will be reliable and stable over relatively long periods of simulation time, allowing the use of SMD to study how these stable structures respond to perturbation and unbinding of the hapten.

\section{Conduction of Ions Through Channels}

In the SMD simulations of the potassium channel, a force directed along the channel axis was applied to a single potas- sium ion to facilitate its passing across the channel. The applied force had the form $\vec{F}(z, t)=K(v t-z) \hat{z}$ (cf. Equation 1), where $z$ is the coordinate of the ion along the channel and $t$ is the elapsed time of the simulation. Since the channel is essentially cylindrically symmetric about its central axis, steering the ion with a unidirectional force directed the ion along nearly the same path as that taken by a free ion. An applied force of this form does not restrain the ion within the plane of the channel. However, if the spring constant is sufficiently stiff, the ion is virtually guaranteed to pass completely through the selectivity filter in time $t=d / v$, where $d \approx 12 \AA$ is the thickness of the selectivity filter.

Results from three simulations (Sim 1, Sim 2a, Sim 2b) will be described. Sim 1 begins with a single ion in the central cavity. Sim 2a and Sim $2 b$ both begin with one ion in the central cavity and one ion at the first binding site, between residues 75 and 76. In Sim 1, the ion was guided through the filter with a restraint moving at a velocity of $20 \AA / n s$. The restraint was held fixed and the system equilibrated for $20 \mathrm{ps}$ before beginning SMD. In Sim 2a and Sim 2b, only the trailing ion (i.e., the ion that began in the central cavity) was restrained. The restraint was moved with velocity $20 \AA / \mathrm{ns}$ in Sim $2 \mathrm{a}$ and $10 \AA / n s$ in Sim $2 b$. In addition, during $\operatorname{Sim} 2 b$, harmonic constraints of strength $10 \mathrm{kcal} / \mathrm{mol} / \AA^{2}$ were applied to the alpha carbons of residues 74-80. These constraints maintain the tertiary structure of the filter while still permitting peptide isomerizations to occur. All three SMD simulations were run at 

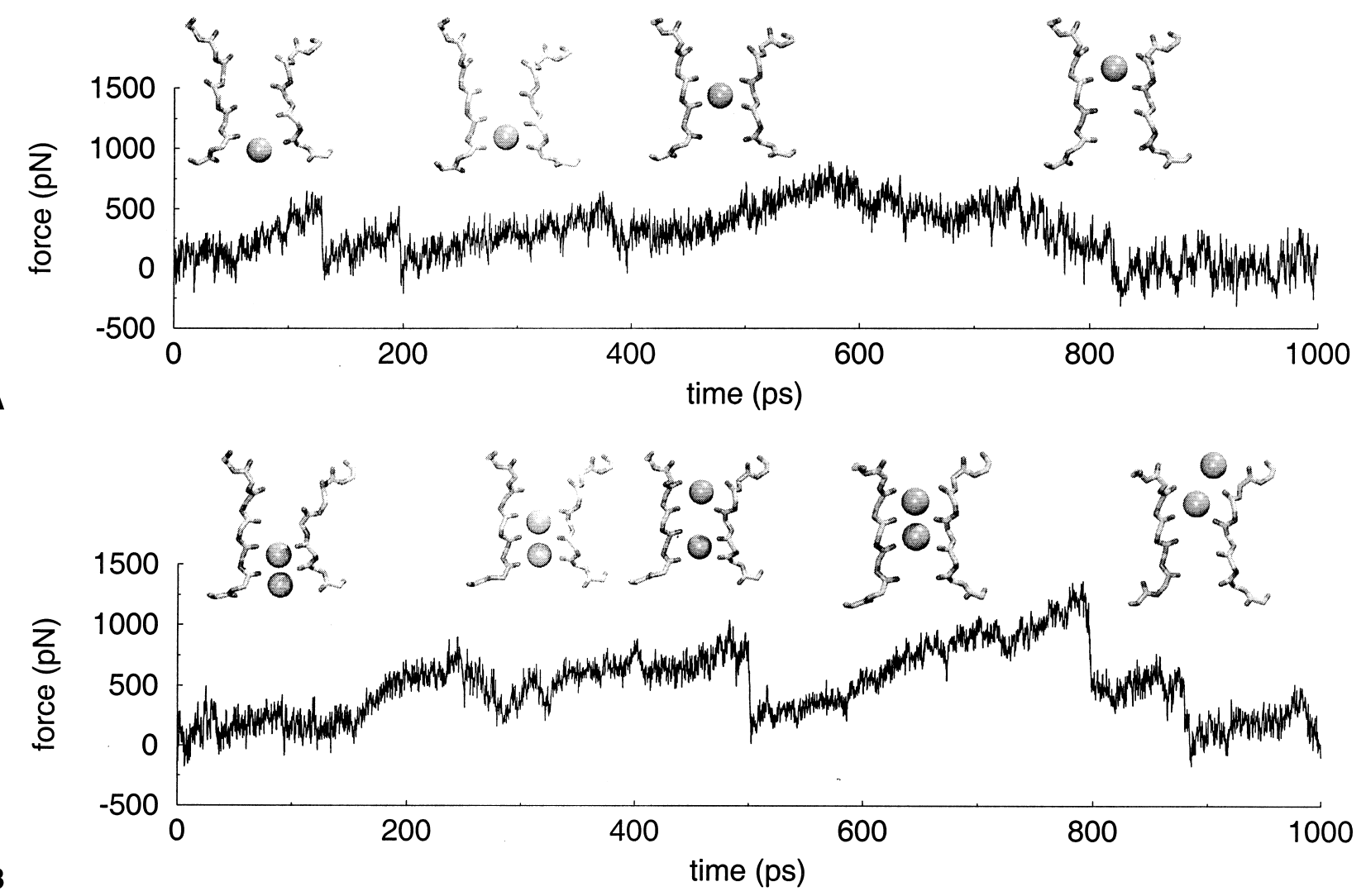

B

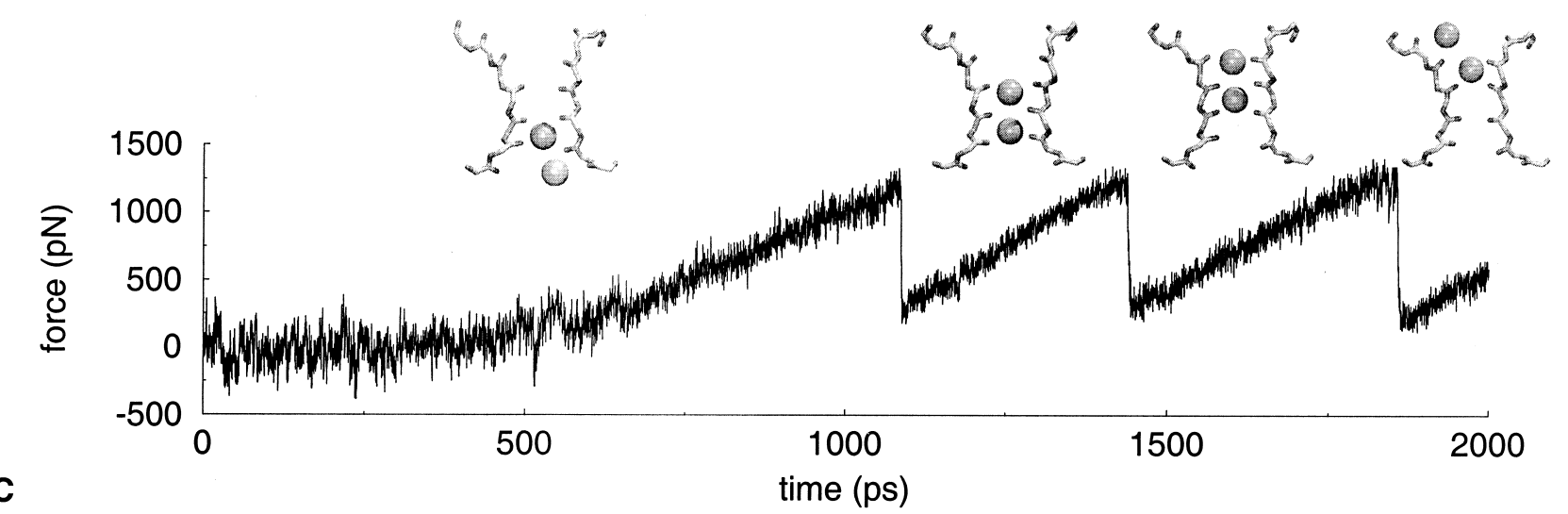

Figure 5. Time evolution of the applied force and of the position of the ion inside the selectivity filter during (a) Sim 1, (b) $\operatorname{Sim} 2 a$, and (c) $\operatorname{Sim} 2 b$.

constant volume and temperature with a damping constant of $10 \mathrm{ps}^{-1}$ to avoid local heating of the channel. The spring constant $K$ was $5.0 \mathrm{kcal} / \mathrm{mol} / \AA^{2}$ in all cases, which corresponds to a thermal mean-square fluctuation of about $2 \AA$.

In the following, PROn, where $n=1,2,3,4$, refers to one of the four subunits of the protein. Elapsed time is measured from the beginning of SMD pulling, i.e., after equilibration of the membrane-protein system and the placement of ions.

Simulation 1: Though the crystal structure ${ }^{55}$ of KcsA showed two potassium ions in the selectivity filter, it is still of interest to compare the dynamics of a single ion in the filter with that of multiple ions. In Sim 1, a single ion was guided through the filter in $1 \mathrm{~ns}$.

The first force peak, as seen in Figure 5a at time 126-133, occurs as the ion moves past the PRO3 carbonyl oxygen over a potential energy barrier, as reflected in the drop in the applied force at $133 \mathrm{ps}$. The ion resides in an energy minimum due to favorable ion-backbone interactions with residue 75 of three of the four subunits.

The second peak in the applied force, at 195-199 ps, occurs as the ion moves into the chamber between $\mathrm{Thr}^{75}$ and $\mathrm{Val}^{76}$. Even at this range, the interactions with $\mathrm{Val}^{76}$ and $\mathrm{Gly}^{77}$ are noticeably stronger, while the interaction with $\mathrm{Thr}^{75}$ remains unchanged.

An interesting peptide isomerization occurs at 380-390 ps, as the PRO3 carbonyl oxygen of $\mathrm{Val}^{76}$ swings around to coordinate the potassium ion in the channel. The ion-backbone interaction energy for PRO3 drops from -15 to $-35 \mathrm{~kJ} / \mathrm{mol}$. A 
later isomerization of the backbone at this site, occurring when the ion is nearly out of the channel, is attributable to interactions with water in and around the filter.

Except for the two early force peaks, there are no sharp features in the force time series. Examination of the trajectory reveals that this is due to the ion traversing the chambers of the four monomers separately; i.e., favorable and unfavorable interactions with backbone atoms of the four monomers are not synchronized. This interpretation is strengthened by an inspection of the ion-backbone interaction energies. Between the sharp jumps in the energies, the time series exhibit a gradual rise, as the monomers that are interacting most strongly with the ion "follow" the ion out until it leaves the chamber altogether.

Simulation 2a: As seen in Figure 5b, the period 239-250 ps corresponds to a gradual transition in which the first ion moves from the second chamber into the third, followed $10 \mathrm{ps}$ later by the trailing ion. Interactions between the trailing ion and $\mathrm{Thr}^{75}$ are constant during this time, while interactions with $\mathrm{Val}^{76}$ and $\mathrm{Gly}^{77}$ become more favorable.

At $400 \mathrm{ps}$, the leading ion moves into the next chamber. There is a small applied force peak at this time, but no drop. The intervening chamber is left unfilled by the trailing ion for approximately 100 ps. At 500 ps, the trailing ion moves into the chamber behind the leading ion, overcoming its attraction to $\mathrm{Thr}^{75}$ and $\mathrm{Val}^{76}$, but moving into a more favorable interaction position with Gly ${ }^{77}$. During this period of 100 ps the interaction with $\mathrm{Val}^{76}$ of PRO3 is rather weak. The trailing ion overcomes an energy barrier of $50 \mathrm{~kJ} / \mathrm{mol}$ due to interactions with the backbone.

Just after the trailing ion moves in, PRO3 isomerizes to bring the carbonyl oxygen of $\mathrm{Gly}^{77}$ into favorable alignment with both potassium ions. The isomerization stabilizes the backbone by $30 \mathrm{~kJ} / \mathrm{mol}$.

At 795-800 ps, the two ions make a concerted transition to the next pair of chambers. Almost simultaneous with this event, the Gly ${ }^{77}$ carbonyl oxygen swings back to its former position. Since the $\mathrm{Gly}^{77}$ oxygen swings away from the ions, the PRO3 interaction drops immediately; the other segments continue to interact strongly until 878 ps elapsed time, when the trailing ion leaves the filter.

Simulation 2b: As seen in Figure 5c, during the first ns the interaction of the trailing ion with $\mathrm{Val}^{76}$ of PRO1 and $\mathrm{Val}^{76}$ of PRO4 increases steadily. After $1,087 \mathrm{ps}$, the two ions make a concerted motion into the next chamber. Very shortly thereafter (5 ps), the $\mathrm{Val}^{76}$ carbonyl oxygen of PRO3 swings around toward the two ions on either side of it. This is similar to the isomerization of $\mathrm{Gly}^{77}$ seen in Sim2a.

A second concerted movement is seen after 1432 ps, accompanied by only a small rotation of $\mathrm{Val}^{76}$ in PRO3, which serves to keep the oxygen closer to the ions. The last movement occurs after $1,854 \mathrm{ps}$, with no major change in the backbone.

Examination of the interaction energies reveals that, between the first and third ion transition events, the trailing ion interacts with all four $\mathrm{Val}^{76}$ residues with equal strength, and equally with three of the four Gly ${ }^{77}$ residues. The force graph (Figure 5c) also shows very sharp, well-defined peaks with quick drops thereafter.

\section{CONCLUSIONS}

Binding and unbinding of noncovalently attached biomolecules are at the heart of many important processes and are the target of experimental investigations. SMD may serve to interpret measurements and suggest new experiments. The rapidly growing computer power available for simulations and increasing time resolution of experimental techniques will provide the basis for further advances in the method and will help bridge the gap in time scales between computer simulation and experiment.

\section{ACKNOWLEDGMENTS}

All figures in this article were created using VMD. ${ }^{51}$ This work was supported by the National Institutes of Health (NIH PHS 5 P41 RR05969, NIH 1 R01 GM60946-01).

\section{REFERENCES}

1 Binning, G., Quate, C.F., and Gerber, G. Atomic force microscope. Phys. Rev. Lett. 1986, 56, 930-933

2 Block, S. and Svoboda, K. Biological applications of optical forces. Ann. Rev. Biophys. Biomol. Struct. 1994, 23, 247-285

3 Evans, E., Ritchie, K., and Merkel, R. Sensitive force technique to probe molecular adhesion and structural linkages at biological interfaces. Biophys. J. 1995, 68, 2580-2587

4 Israelachvili, J.N. Intermolecular and Surface Forces. Academic Press, London, 1992

5 Izrailev, S., Stepaniants, S., Balsera, M., Oono, Y., and Schulten, K. Molecular dynamics study of unbinding of the avidin-biotin complex. Biophys. J. 1997, 72, 15681581

6 Isralewitz, B., Izrailev, S., and Schulten, K. Binding pathway of retinal to bacterio-opsin: A prediction by molecular dynamics simulations. Biophys. J. 1997, 73, 2972-2979

7 Wriggers, W. and Schulten, K. Investigating a back door mechanism of actin phosphate release by steered molecular dynamics. Proteins: Struct. Func., and Genetics 1999, 35, 262-273

8 Kosztin, D., Izrailev, S., and Schulten, K. Unbinding of retinoic acid from its receptor studied by steered molecular dynamics. Biophys. J. 1999, 76, 188-197

9 Stepaniants, S., Izrailev, S., and Schulten, K. Extraction of lipids from phospholipid membranes by steered molecular dynamics. J. Mol. Model. 1997, 3, 473-475

10 Molnar, F., Ben-Nun, M., Martínez, T.J., and Schulten, $\mathrm{K}$. Characterization of a conical intersection between the ground and first excited state for a retinal analog. J. Mol. Struct. (THEOCHEM), WATOC special issue 2000, 506, $169-178$

11 Lu, H., Isralewitz, B., Krammer, A., Vogel, V., and Schulten, K. Unfolding of titin immunoglobulin domains by steered molecular dynamics simulation. Biophys. J. 1998, 75, 662-671

12 Krammer, A., Lu, H., Isralewitz, B., Schulten, K., and Vogel, V. Forced unfolding of the fibronectin type III module reveals a tensile molecular recognition switch. Proc. Natl. Acad. Sci. U.S.A. 1999, 96, 1351-1356

$13 \mathrm{Lu}, \mathrm{H}$. and Schulten, K. Steered molecular dynamics simulations of force-induced protein domain unfolding. 
Proteins: Struct., Func., and Genetics 1999, 35, 453463

$14 \mathrm{Lu}, \mathrm{H}$. and Schulten, K. Steered molecular dynamics simulation of conformational changes of immunoglobulin domain I27 interpret atomic force microscopy observations. Chem. Phys. 1999, 247, 141-153

15 Izrailev, S., Stepaniants, S., Isralewitz, B., Kosztin, D., Lu, H., Molnar, F., Wriggers, W., and Schulten, K. Steered molecular dynamics. In: Deuflhard, P., Hermans, J., Leimkuhler, B., Mark, A.E., Reich, S., and Skeel, R.D., Eds., Computational Molecular Dynamics: Challenges, Methods, Ideas, volume 4 of Lecture Notes in Computational Science and Engineering, SpringerVerlag, Berlin, 1998, pp. 39-65

16 Grubmüller, H., Heymann, B., and Tavan, P. Ligand binding and molecular mechanics calculation of the streptavidin-biotin rupture force. Science 1996, 271, 997-999

17 Schlitter, J., Engels, M., Krüger, P., Jacoby, E., and Wollmer, A. Targeted molecular dynamics simulation of conformational change - application to the $\mathrm{T} \rightarrow \mathrm{R}$ transition in insulin. Molecular Simulation 1993, 10(2-6), 291-308

18 Marrink, S.-J., Berger, O., Tieleman, P., and Jähnig, F. Adhesion forces of lipids in a phospholipid membrane studied by molecular dynamics simulations. Biophys. J. 1998, 74, 931-943

19 Wade, R.C., Gabdoulline, R.R., Ludemann, S.K., and Lounnas, V. Electrostatic steering and ionic tethering in enzyme-ligand binding: Insights from simulations. Proc. Natl. Acad. Sci. U.S.A. 1998, 95, 5942-5949

20 Paci, E. and Karplus, M. Forced unfolding of fibronectin type 3 modules: An analysis by biased molecular dynamics simulations. J. Mol. Biol. 1999, 288, 441-459

21 Pain, R.H. Mechanisms of Protein Folding. Oxford University Press, New York, 1994

22 Onuchic, J.N., Luthey-Schulten, Z., and Wolynes, P.G. Theory of protein folding: The energy landscape perspective. Annu. Rev. Phys. Chem. 1997, 48, 545-600

23 Brooks, C. Simulations of protein folding and unfolding. Curr. Opinion Struct. Biol. 1998, 8, 222-226

24 Duan, Y. and Kollman, P. Pathways to a protein folding intermediate observed in a 1 microsecond simulation in aqueous solution. Science 1998, 282, 740-744

25 Gruebele, M., Sabelko, J., Ballew, R., and Ervin, J. Laser T-jump induced protein re-folding. Accts. Chem. Res. 1998, 31, 699-707

26 Pande, V., Grosberg, A., Tanaka, T., and Rokhsar, D. Pathways for protein folding: is a new view needed? Curr. Opinion Struct. Biol. 1998, 8, 68-79

27 Dill, K.A. and Chan, H.S. From levinthal to pathways to funnels. Nature Struct. Biol. 1997, 4, 10-19

28 Tirado-Rives, J., Orozco, M., and Jorgensen, W. Molecular dynamics simulations of the unfolding of barnase in water and 8M aqueous urea. Biochemistry 1997, 36, 7313-7329

29 Rothwarf, D. and Scheraga, H.A. Role of non-native aromatic and hydrophobic interactions in the folding of hen egg white lysozyme. Biochemistry 1998, 35, 1379713807

30 Hunenberger, P.H., Mark, A.E., and van Gunsteren, W. Computational approaches to study protein unfolding: Hen egg white lysozyme as a case study. Proteins: Struc., Func., Genetics 1995, 21, 196-213
31 Karplus, M. and Sali, A. Theoretical studies of protein folding and unfolding. Curr. Opinion Struct. Biol. 1995, 5, 58-73

32 Dagget, V. and Levitt, M. Protein folding-unfolding dynamics. Curr. Opinion Struct. Biol. 1994, 4, 291-295

33 Rief, M., Gautel, M., Oesterhelt, F., Fernandez, J.M., and Gaub, H.E. Reversible unfolding of individual titin immunoglobulin domains by AFM. Science 1997, 276, 1109-1112

34 Kellermayer, M., Smith, S., Granzier, H., and Bustamante, C. Folding-unfolding transition in single titin modules characterized with laser tweezers. Science 1997, 276, 1112-1116

35 Tskhovrebova, L., Trinick, J., Sleep, J., and Simmons, R. Elasticity and unfolding of single molecules of the giant protein titin. Nature 1997, 387, 308-312

36 Oberhauser, A.F., Marszalek, P.E., Erickson, H., and Fernandez, J. The molecular elasticity of tenascin, an extracellular matrix protein. Nature 1998, 393, 181-185

37 Rief, M., Gautel, M., Schemmel, A., and Gaub, H. The mechanical stability of immunoglobulin and fibronectin III domains in the muscle protein titin measured by afm. Biophys. J. 1998, 75, 3008-3014

38 Maruyama, K. Connectin/titin, a giant elastic protein of muscle. FASEB J. 1997, 11, 341-345

39 Wang, N., Butler, J.P., and Ingber, D.E. Mechanotransduction across the cell surface and through the cytoskeleton. Science 1993, 260, 1124-1127

40 Machado, C., Sunkel, C., and Andrew, D. Human autoantibodies reveal titin as a chromosomal protein. J. Cell. Biol. 1998, 141, 321-333

41 Rief, M., Pascual, J., Saraste, M., and Gaub, H. Single molecule force spectroscopy of spectrin repeats: Low unfolding forces in helix bundles. J. Mol. Biol. 1999, 286, 553-561

42 Leech, J., Prins, J., and Hermans, J. SMD: Visual steering of molecular dynamics for protein design. IEEE Comp. Sci. Eng. 1996, 3(4), 38-45

43 Rohs, R., Etchebest, C., and Lavery, R. Unraveling proteins: A molecular mechanics study. Biophys. J. 1999, 76, 2760-2768

44 Socci, N., Onuchic, J., and Wolynes, P. Stretching lattice models of protein folding. Proc. Natl. Acad. Sci. U.S.A. 1999, 96, 2031-2035

45 Evans, E. and Ritchie, K. Strength of a weak bond connecting flexible polymer chains. Biophys. J. 1999, 76, 2439-2447

46 Persidis, A. Catalytic antibodies. Some companies are taking an active interest in this promising technology. Nature Biotechnology 1997, 15, 1313-1315

47 French, D.L., Laskov, R., and Scharff, M.D. The role of somatic hypermutation in the generation of antibody diversity. Science 1989, 244, 1152-1157

48 Patten, P., Gray, N., Yang, P., Marks, C., Wedemayer, G., Boniface, J., Stevens, R., and Schultz, P. The immunological evolution of catalysis. Science 1996, 271, 1086-1091

49 Wedemeyer, G., Wang, L., Patten, P., Schultz, P., and Stevens, R. Crystal structure of the free and liganded form of an esterolytic catalytic antibody. J. Mol. Biol. 1997, 268, 390-400

50 Wedemeyer, G., Patten, P., Wang, L., Schultz, P., and Stevens, R. Structural insights into the evolution of an antibody combining site. Science 1997, 276, 1665-1669 
51 Humphrey, W.F., Dalke, A., and Schulten, K. VMDVisual Molecular Dynamics. J. Mol. Graphics 1996, 14, 33-38

52 Pomès, R., Willson, R.C., and McCammon, J.A. Free energy simulations of the HyHEL-10/HEL antibodyantigen complex. Protein Engineering 1995, 3(7), 663675

53 Lim, K. and Herron, J.N. Molecular dynamics of the anti-fluorescein 4-4-20 antigen-binding fragment. 1. computer simulations. Biochemistry 1995, 34, 69626974

54 Hille, B. Ionic Channels of Excitable Membranes, 2nd edition, Sinauer Associates, Sunderland, MA, 1992

55 Doyle, D.A., Cabral, J.M., Pfuetzer, R.A., Kuo, A., Gulbis, J.M., Cohen, S.L., Chait, B.T., and MacKinnon, R. The structure of the potassium channel: molecular basis of $\mathrm{K}^{+}$conduction and selectivity. Science 1998, 280, 69-77

56 Heginbotham, L., Lu, Z., Abramson, T., and MacKinnon, R. Mutations in the $\mathrm{K}^{+}$channel signature sequence. Biophys. J. 1994, 64, 1061-1067

57 Young, M.A., Ravishanker, G., and Beveridge, D.L. A 5-nanosecond molecular dynamics trajectory for B-DNA: analysis of structure, motion and solvation. Biophys. J. 1997, 73, 2313-2336

58 Feig, M. and Pettitt, B.M. Modeling high resolution hydration patterns in correlation with DNA sequence and conformation. J. Mol. Biol. 1999, 286, 1075-1095

59 Gullingsrud, J., Braun, R., and Schulten, K. Reconstructing potentials of mean force through time series analysis of steered molecular dynamics simulations. J. Comp. Phys. 1999, 151, 190-211

60 McCammon, J.A. and Harvey, S.C. Dynamics of Proteins and Nucleic Acids. Cambridge University Press, Cambridge, 1987

61 Kumar, S., Bouzida, D., Swendsen, R.H., Kollman, P.A., and Rosenberg, J.M. The weighted histogram analysis method for free-energy calculations on biomolecules. I. The method. J. Comp. Chem. 1992, 13, 1011-1021

62 Jarzynski, C. Nonequilibrium equality for free energy differences. Phys. Rev. Lett. 1997, 78, 2690-2693

63 Jarzynski, C. Equilibrium free-energy differences from nonequilibrium measurements: A master equation approach. Phys. Rev. E 1997, 56, 5018-5035

64 Balsera, M., Stepaniants, S., Izrailev, S., Oono, Y., and Schulten, K. Reconstructing potential energy functions from simulated force-induced unbinding processes. Biophys. J. 1997, 73, 1281-1287

65 Marszalek, P.E., Lu, H., Li, H., Carrion-Vasquez, M.,
Oberhauser, A.F., Schulten, K., and Fernandez, J.M. Mechanical unfolding intermediates in titin modules. Nature 1999, 402, 100-103

66 Wriggers, W. and Schulten, K. Protein domain movements: Detection of rigid domains and visualization of hinges in comparisons of atomic coordinates. Proteins: Struc., Func., and Genetics 1997, 29(1), 1-14

67 Lüdemann, S.K., Carugo, O., and Wade, R.C. Substrate access to cytochrome P450cam: A comparison of a thermal motion pathway analysis with molecular dynamics simulation data. J. Mol. Model 1997, 3, 369-374

68 Baljon, R.C.A. and Robbins, M.O. Energy dissipation during rupture of adhesive bonds. Science 1996, 271, 482-484

$69 \mathrm{Xu}$, D., Phillips, J.C., and Schulten, K. Protein response to external electric fields: Relaxation, hysteresis and echo. J. Phys. Chem. 1996, 100, 12108-12121

70 Roux, B. The calculation of the potential of mean force using computer simulations. Computer Physics Communications 1995, 91, 275-282

71 Gardiner, C.W. Handbook of Stochastic Methods for Physics, Chemistry, and the Natural Sciences. SpringerVerlag, New York, 1985

72 van Kampen, N.G. Stochastic Processes in Physics and Chemistry. North-Holland, Amsterdam, New York, 1992

73 Onsager, L. and Machlup, S. Fluctuations and irreversible processes. Phys. Rev. 1953, 91, 1505-1512

74 Zanetti, M., Filaci, G., Lee, R., del Guercio, P., Rossi, F., Bacchetta, R., Stevenson, F., Barnaba, V., and Billetta, R. Expression of conformationally constrained adhesion peptide in an antibody CDR loop and inhibition of natural killer cell cytotoxic activity by an antibody antigenized with the RGD motif. EMBO J. 1993, 12, 4375-4384

75 Beer, J., Springer, K., and Coller, B. Immobilized arggly-asp (RGD) peptides of varying lengths as structural probes of the platelet glycoprotein IIb/IIIa receptor. Blood 1992, 79(1), 117-128

76 Brooks, B.R., Bruccoleri, R.E., Olafson, B.D., States, D.J., Swaminathan, S., and Karplus, M. CHARMM: A program for macromolecular energy, minimization, and dynamics calculations. J. Comp. Chem. 1983, 4, 187217

77 Kalé, L., Skeel, R., Bhandarkar, M., Brunner, R., Gursoy, A., Krawetz, N., Phillips, J., Shinozaki, A., Varadarajan, K., and Schulten, K. NAMD2: Greater scalability for parallel molecular dynamics. J. Comp. Phys. 1999, 151, 283-312 